\title{
Semigroups on Frechet spaces and equations with infinite delays
}

\section{T SENGADIR}

Department of Mathematics, SSN College of Engineering, Old Mahabalipuram Road, Kalavakkam 603 110, India

MS received 28 February 2005

\begin{abstract}
In this paper, we show existence and uniqueness of a solution to a functional differential equation with infinite delay. We choose an appropriate Frechet space so as to cover a large class of functions to be used as initial functions to obtain existence and uniqueness of solutions.
\end{abstract}

Keywords. Functional differential equation; infinite delay; semigroup; Frechet space.

\section{Introduction and preliminaries}

In this paper we study linear functional differential equations with infinite delay. Consider

$$
\begin{aligned}
& x^{\prime}(t)=\operatorname{ax}(t)+\sum_{i=1}^{\infty} b_{i} x\left(t-\tau_{i}\right), \quad t \geq 0 \\
& x(\theta)=\phi(\theta), \quad \theta \in(-\infty, 0]
\end{aligned}
$$

where $a \in \mathbb{R},\left\{b_{i}\right\}_{i=1}^{\infty}$ is an arbitrary sequence of real numbers, $\left\{\tau_{i}\right\}_{i=1}^{\infty}$ is a strictly increasing sequence of strictly positive reals such that $\lim _{i \rightarrow \infty} \tau_{i}=\infty$ and $\phi:(-\infty, 0] \longrightarrow \mathbb{R}$ is continuous.

For the special case $\left\{b_{i}\right\}_{i=1}^{\infty} \in l^{1},(1.1)$ can be uniquely solved for any given $\phi \in \mathrm{BC}$ $(-\infty, 0]$, the space of all bounded real-valued continuous functions. The proof of this is indicated in Example1.2. Denote this solution by $x_{\phi}$. Consider the family of operators $S_{t}, t \geq 0$ on BC $(-\infty, 0]$ defined as

$$
\begin{aligned}
{\left[S_{t} \phi\right](\theta) } & =x_{\phi}(t+\theta), \quad \text { if } t+\theta>0 \\
& =\phi(t+\theta), \quad \text { if } t+\theta \leq 0 .
\end{aligned}
$$

It is elementary to see that $\left\{S_{t}\right\}$ is not a strongly continuous semigroup on BC $(-\infty, 0]$. We prove this as follows: If $S_{t}$ is a semigroup, we must have

$$
\lim _{t \rightarrow 0} S_{t} \phi=\phi
$$

Given $\epsilon>0$, we can find $\delta>0$ such that $\left\|S_{t} \phi-\phi\right\|_{\infty} \leq \epsilon$ for $|t| \leq \delta$. Now, let $\delta^{*}=\min (1, \delta)$. Consider $\theta_{1}, \theta_{2} \in(-\infty,-1]$ with $0 \leq \theta_{2}-\theta_{1} \leq \delta^{*}$. Let $t=\theta_{2}-\theta_{1}$. Note that $0 \leq t \leq \delta$ and

$$
\theta_{1}+t \leq \theta_{2}+\delta \leq \theta_{2}+1 \leq 0 \text {. }
$$


Consider

$$
\begin{aligned}
\left|\phi\left(\theta_{2}\right)-\phi\left(\theta_{1}\right)\right| & \leq \mid \phi\left(t+\theta_{1}\right)-\phi\left(\theta_{1} \mid\right. \\
& \leq\left|\left(S_{t} \phi\right)\left(\theta_{1}\right)-\phi\left(\theta_{1}\right)\right| \\
& \leq\left\|S_{t} \phi-\phi\right\|_{\infty} \\
& \leq \epsilon .
\end{aligned}
$$

Thus, we have shown that $\phi$ is uniformly continuous on $(-\infty,-1]$. By the uniform continuity of $\phi$ on $[-1,0]$, uniform continuity of $\phi$ on $(-\infty, 0]$ follows. But this is a contradiction as there are bounded continuous functions which are not uniformly continuous.

On the space BUC $(-\infty, 0]$, the space of bounded uniformly continuous functions, the family of operators $S_{t}$ defined by (1.2) do form a semigroup but this space is properly contained in $\mathrm{BC}(-\infty, 0]$. In the literature, certain Banach spaces, which contain $\mathrm{BC}(-\infty, 0]$, which are classes of functions satisfying certain growth conditions, are used in the context of infinite delay equations. Refer $[5,6]$. Our approach is to find a Frechet space that contains $\mathrm{BC}(-\infty, 0]$ on which $S_{t}$ 's form a semigroup.

Instead of constructing a weight function which is related to $b_{i}$ and $\tau_{i}$, we obtain a family of semi-norms for the initial function $\phi$ that enable us to get estimates for the solution and also to capture a Frechet space $F$ such that (1.2) defines a strongly continuous semigroup on $F$. We do not make any explicit summability assumption on the sequence $\left\{b_{i}\right\}_{i=1}^{\infty}$, but the space $F$ heavily depends on the properties of $\left\{b_{i}\right\}_{i=1}^{\infty}$. If $\left\{b_{i}\right\} \in l^{1}$, then $\mathrm{BC}(-\infty, 0] \subset F$ but if $b_{i}=\frac{1}{i}, \mathrm{BC}(-\infty, 0]$ is not contained in $F$.

The basic theory of finite delay differential equations is covered in [7]. [8] and [1] are some basic references for Banach phase spaces related to infinite delay equations. Consider the following examples:

Example 1.1. Let $X=\mathrm{BC}[0, \infty)$ and $A: \mathcal{D}(A)=\mathrm{BC}^{1}[0, \infty) \longrightarrow \mathrm{BC}[0, \infty)$ be defined as $[A \varphi](x)=\varphi^{\prime}(x)$. Define $S_{t}: X \longrightarrow X, t \geq 0$, as $\left(S_{t} \varphi\right)(x)=\varphi(t+x)$. It is easy to see that $A$ satisfies the following conditions:

(a) the resolvents $(\lambda I-A)^{-1} u=\int_{0}^{\infty} \mathrm{e}^{-\lambda \tau} S_{\tau} u \mathrm{~d} \tau$ exist for $\lambda>0$, where $I$ is the identity operator on $X$,

(b) $\left\|(\lambda I-A)^{-1}\right\| \leq \frac{1}{\lambda}$ and that $S_{t}$ satisfy the following conditions:

(i) $S_{0}=I$,

(ii) $S_{t+s}=S_{t} S_{s}$.

Despite the above observations $S_{t}$ is not strongly semigroup on $X$ since the condition

(iii) $\lim _{t \rightarrow 0} S_{t} \varphi=\varphi, \varphi \in X$ does not hold.

The proof that (iii) does not hold is similar to the proof of the analogous assertion in the case of the infinite delay equations. The Hille-Yosida theorem is not applicable precisely because $A$ is not densely defined. One way to overcome this difficulty is to construct a smaller Banach space called the Hille-Yosida space for the operator $A[10]$ on which the restriction of $A$ generates a semigroup. In this construction, the condition (a) plays a crucial role.

But consider the operator $\mathcal{A}: \mathcal{D}(\mathcal{A})=\mathbf{C}^{1}[0, \infty) \longrightarrow \mathbf{C}[0, \infty)$ defined as $[\mathcal{A} \varphi](x)=$ $\varphi^{\prime}(x) . \mathcal{A}$ is an 'extension' of $A$ that does generate the semigroup $\mathcal{S}_{t}: \mathbf{C}[0, \infty) \longrightarrow \mathbf{C}[0, \infty)$ 
defined as $\left[\mathcal{S}_{t}\right] \varphi(x)=\varphi(t+x)$ on the Frechet space $\mathbf{C}[0, \infty)$. Thus, it is clear that by considering the Hille-Yosida space, a lot of useful information is lost.

Example 1.2. We now indicate the proof of assertion that for $\left\{b_{i}\right\} \in l^{1}$, (1.1) can be uniquely solved for any given $\phi \in \mathrm{BC}(-\infty, 0]$.

For $t \in\left[0, \tau_{1}\right], t-\tau_{i} \in(-\infty, 0]$ and hence for $\phi \in \mathrm{BC}(-\infty, 0], \phi\left(t-\tau_{i}\right)$ is meaningful. Define $z_{i} \in C\left[0, \tau_{1}\right]$ as $z_{i}=b_{i} \phi\left(t-\tau_{i}\right)$. Now $\left\|z_{i}\right\|_{\infty}=\left|b_{i}\right| \sup _{t \in\left[0, \tau_{1}\right]}\left|\phi\left(t-\tau_{i}\right)\right| \leq$ $\left|b_{i}\right|\|\phi\|_{\infty}=\left|b_{i}\right|\|\phi\|_{\infty}$. Since $\left\{b_{i}\right\}$ is in $l^{1}$, the series $\sum_{i=1}^{\infty}\left\|z_{i}\right\|$ converges. Hence $\sum_{i=1}^{\infty} z_{i}$ converges in $C\left[0, \tau_{1}\right]$. Thus, $\sum_{i=1}^{\infty} \phi\left(t-\tau_{i}\right) \in C\left[0, \tau_{1}\right]$. Now, consider the ordinary differential equation

$$
\begin{aligned}
& x^{\prime}(t)=\operatorname{ax}(t)+\sum_{i=1}^{\infty} b_{i} \phi\left(t-\tau_{i}\right), \quad t \in\left[0, \tau_{1}\right], \\
& x(0)=\phi(0)
\end{aligned}
$$

whose solution exists and is unique.

Now, assume the existence of a unique function $y_{k}:\left(-\infty, k \tau_{1}\right] \longrightarrow \mathbb{R}$ such that $y_{k}(\theta)=$ $\phi(\theta)$ for $\theta \in(-\infty, 0]$ and whose restriction to $\left[0, k \tau_{1}\right]$ is a solution to

$$
\begin{aligned}
& x^{\prime}(t)=a x(t)+\sum_{i=1}^{\infty} b_{i} x\left(t-\tau_{i}\right), \quad t \in\left[0, k \tau_{1}\right], \\
& x(0)=\phi(0) .
\end{aligned}
$$

As before, the expression $\sum_{i=1}^{\infty} b_{i} y_{k}\left(t-\tau_{i}\right)$ defines a continuous function on $\left[k \tau_{1},(k+\right.$ 1) $\tau_{1}$ ]. Now, consider

$$
\begin{aligned}
x^{\prime}(t) & =a x(t)+\sum_{i=1}^{\infty} b_{i} y_{k}\left(t-\tau_{i}\right), \quad t \in\left[k \tau_{1},(k+1) \tau_{1}\right], \\
x\left(k \tau_{1}\right) & =y_{k}\left(k \tau_{1}\right)
\end{aligned}
$$

which has a unique solution $z$. Define $y_{k+1}$ as $y_{k+1}(s)=y_{k}(s), s \in\left(-\infty, k \tau_{1}\right]$ and $y_{k+1}(s)=z(s), s \in\left[k \tau_{1},(k+1) \tau_{1}\right]$. Thus, we obtain a solution to $(1.1)$ in $\left(-\infty,(k+1) \tau_{1}\right]$. By induction and patching up of solutions, we get a unique solution to (1.1) on the whole of $(-\infty, \infty)$.

Let $X=B C(-\infty, 0]$ and $\left\{b_{i}\right\}_{i=1}^{\infty} \in l^{1}$. Define

$$
A: \mathcal{D}(A)=\left\{\varphi \in \mathrm{BC}^{1}(-\infty, 0]: \varphi^{\prime}(0)=\sum_{i=1}^{\infty} b_{i} \varphi\left(-\tau_{i}\right)\right\} \longrightarrow \mathrm{BC}(-\infty, 0]
$$

as

$$
[A \varphi](x)=\varphi^{\prime}(x)
$$

$\mathcal{D}(A)$ is not dense in $X$ and hence $A$ does not generate a semigroup. Motivated by Example 1.1 , we look for a Frechet space $F$ that contains $\mathrm{BC}(-\infty, 0]$ and an 'extension' $\mathcal{A}$ to $A$ such that $\mathcal{A}$ generates a semigroup on $F$. 
Remark 1.3. The general theory of semigroups on Frechet spaces is very complicated. For example, even a bounded linear operator on a Frechet space need not generate a semigroup [12]. In [11], a generalisation of the Hille-Yosida theorem for a closed and unbounded operator in a locally convex space is proved. But the hypotheses of this theorem are not easily verified in many concrete cases. By proving various estimates for the solution $x$ of (1.1), we are able to capture a Frechet space $F$ on which the solution of (1.1) gives rise to a semigroup.

Refer to [3], [13] and [2] for applications of semigroups on locally convex spaces to PDE's.

We need the following definitions and results in the next section.

\section{DEFINITION 1.4}

(i) A topological vector space $X$ is said to be a Frechet space if its topology is generated by a family of countable semi-norms $\left\{q_{i}\right\}_{i=1}^{\infty}$ and $X$ is complete with respect to the family $\left\{q_{i}\right\}_{i=1}^{\infty}$.

(ii) A linear map $S: X \rightarrow X$ is said to be bounded if for every $i \in \mathbb{N}$, there are finitely many indices $j_{1}, j_{2}, \ldots, j_{m}$ and a constant $C$ such that for all $\phi \in X$,

$$
q_{i}(S \phi) \leq C \max \left(q_{j_{1}}(\phi), q_{j_{2}}(\phi), \ldots, q_{j_{m}}(\phi)\right) .
$$

The basic theory of Frechet spaces and the proof of the following proposition can be found in [14].

\section{PROPOSITION 1.5}

A linear map $S: X \rightarrow X$ is continuous if and only if it is bounded.

\section{DEFINITION 1.6}

A family of bounded linear operators $\left\{S_{t}: t \geq 0\right\}$ on $X$ is said to be a strongly continuous semigroup if the properties (i), (ii) and (iii) of Example 1.1 hold.

\section{PROPOSITION 1.7}

Let $l^{1}(X)$ denote the Banach space of all sequences $\left\{x_{i}\right\}_{i=1}^{\infty}$ of elements of a Banach $X$ such that $\sum_{i=1}^{\infty}\left\|x_{i}\right\|<\infty$. Let $\mathbf{C}\left([0, T] ; l^{1}\right)$ be the Banach space of all continuous functions $h:[0, T] \longrightarrow l^{1}$. The Banach space $l^{1}(\mathbf{C}([0, T]))$ is isometrically embedded in $\mathbf{C}\left([0, T] ; l^{1}\right)$.

Proof. Let $G \in l^{1}(\mathbf{C}([0, T]))$. Define $G^{*}:[0, T] \longrightarrow l^{1}(\mathbb{R})$ as $\left[G^{*}(s)\right]^{(i)}=G^{(i)}(s)$. It is given that

$$
\sum_{i=1}^{\infty} \sup \left\{\left|G^{(i)}(s)\right|: s \in[0, T]\right\}<\infty .
$$

It is easy to check that $G^{*}$ is a bounded function and that

$$
\sup \left\{\sum_{i=1}^{\infty}\left|\left[G^{*}(s)\right]^{(i)}\right|: s \in[0, T]\right\}=\sum_{i=1}^{\infty} \sup \left\{\left|G^{(i)}(s)\right|: s \in[0, T]\right\} .
$$


It remains to be shown that

$$
\lim _{t \rightarrow t_{0}} \sum_{i=1}^{\infty}\left|\left[G^{*}(t)\right]^{(i)}-\left[G^{*}\left(t_{0}\right)\right]^{(i)}\right|=0 .
$$

By the hypothesis for each $\left.i, \lim _{t \rightarrow t_{0}} \mid\left[G^{*}(t)\right]^{(i)}-G^{*}\left(t_{0}\right)\right]^{(i)} \mid=0$ and for a given $\epsilon>0$, there exists $K \in \mathbb{N}$ such that

$$
\sum_{i=K+1}^{\infty} \sup \left\{\left|G^{(i)}(s)\right|: s \in[0, T]\right\}<\epsilon / 3 .
$$

Further, there exists $\delta>0$ such that $\left|t-t_{0}\right|<\delta$ implies that

$$
\sum_{i=1}^{K}\left|\left[G^{*}(t)\right]^{(i)}-\left[G^{*}\left(t_{0}\right)\right]^{(i)}\right|<\epsilon / 3 .
$$

Hence for all $t, t_{0}$ in $[0, T]$ with $\left|t-t_{0}\right|<\delta$,

$$
\begin{aligned}
\sum_{i=1}^{\infty}\left|\left[G^{*}(t)\right]^{[(i)}-\left[G^{*}\left(t_{0}\right)\right]^{[(i)}\right|= & \sum_{i=1}^{K}\left|\left[G^{*}(t)\right]^{[(i)}-\left[G^{*}\left(t_{0}\right)\right]^{[(i)}\right| \\
& +\sum_{i=K+1}^{\infty}\left|\left[G^{*}(t)\right]^{[(i)}-\left[G^{*}\left(t_{0}\right)\right]^{[(i)}\right| \leq \epsilon / 3 \\
& +\sum_{i=K+1}^{\infty}\left|\left[G^{*}(t)\right]^{[(i)}\right|+\sum_{i=K+1}^{\infty}\left|\left[G^{*}\left(t_{0}\right)\right]^{[(i)}\right| \\
\leq & \epsilon / 3+\epsilon / 3+\epsilon / 3=\epsilon .
\end{aligned}
$$

The result is proved.

The following definitions and statements on Frechet space valued Riemann integral are found in [9].

Theorem 1.8. Let $X$ be a Frechet space and let $u:[a, b] \longrightarrow X$ be continuous. The integral $\int_{a}^{b} u(t) \mathrm{d} t \in X$ can be defined uniquely which has the following properties:

(i) for every continuous linear functional $x^{*}: X \longrightarrow \mathbb{R}$,

$$
x^{*}\left(\int_{a}^{b} u(t) \mathrm{d} t\right)=\int_{a}^{b} x^{*}(u(t)) \mathrm{d} t,
$$

(ii) for every seminorm $q_{k}$,

$$
q_{k}\left(\int_{a}^{b} u(t) \mathrm{d} t\right) \leq \int_{a}^{b} q_{k}(u(t)) \mathrm{d} t,
$$

(iii) $\int_{a}^{b} u(t) \mathrm{d} t+\int_{b}^{c} u(t) \mathrm{d} t=\int_{a}^{c} u(t) \mathrm{d} t$ 
(iv) $\int_{a}^{b}[u(t)+v(t)] \mathrm{d} t=\int_{a}^{b} u(t) \mathrm{d} t+\int_{a}^{b} v(t) \mathrm{d} t$,

(v) $c \int_{a}^{b} u(t) \mathrm{d} t=\int_{a}^{b} c u(t) \mathrm{d} t$.

\section{DEFINITION 1.9}

Let $J$ be a sub interval of $\mathbb{R}$. A function $u: J \longrightarrow X$ is said to be differentiable at $t_{0} \in J$ if there exists $y \in X$ with the following property: for every $\epsilon>0$ and $k \in \mathbb{N}$, there exists $\delta>0$ such that for all $h \in \mathbb{R}$ with $\left|t_{0}+h\right|<\delta$ and $t_{0}+h \in J$, we have

$$
q_{k}\left(\frac{u\left(t_{0}+h\right)-u\left(t_{0}\right)}{h}-y\right)<\epsilon .
$$

Further, the element $y$ is denoted by $u^{\prime}\left(t_{0}\right)$.

\section{DEFINITION 1.10}

Let $J$ be a sub interval of $\mathbb{R}$. A function $u: J \longrightarrow X$ is said to be continuously differentiable on $J$, if $u$ is differentiable at every point $t_{0} \in J$ and the function mapping $t$ to $u^{\prime}(t)$ is continuous on $J$. The class of all such functions is denoted by $\mathbf{C}^{1}(J ; X)$.

\section{Fundamental theorem of integral calculus}

If $u:[a, b] \longrightarrow X$ is continuously differentiable, then

$$
u(a)-u(b)=\int_{a}^{b} u^{\prime}(t) \mathrm{d} t .
$$

A Frechet phase space

For a given $\varphi \in \mathbf{C}((-\infty, 0])$ define the family of seminorms $\left\{\|\cdot\|_{k}: k \in \mathbb{N}\right\}$ as

$$
\|\varphi\|_{k}=\sup \{|\varphi(\theta)|: \theta \in[-k, 0]\} .
$$

Let $\left\{b_{i}\right\}_{i=1}^{\infty}$ and $\left\{\tau_{i}\right\}_{i=1}^{\infty}$ be as in the beginning of this section. Given $k \in \mathbb{N}$, define $n(k) \in \mathbb{N}$ as the smallest positive integer such that $\tau_{i} \geq k \tau_{1}$ for all $i \geq n(k)$.

Define $F$ as

$$
F=\left\{\varphi \in \mathbf{C}(-\infty, 0]: p_{k}(\varphi)<\infty \text { for all } k \in \mathbb{N}\right\},
$$

where the seminorms $p_{k}$ are defined as follows:

$$
p_{k}(\varphi)=\sum_{i=n(k)}^{\infty} \sup \left\{\left|b_{i} \varphi\left(s-\tau_{i}\right)\right|: s \in\left[0, k \tau_{1}\right]\right\} .
$$

\section{PROPOSITION 1.11}

The space $F$ equipped with the topology generated by the family of seminorms $\left\{\|\cdot\|_{k}: k \in\right.$ $\mathbb{N}\} \cup\left\{p_{k}: k \in \mathbb{N}\right\}$ is a Frechet space. 
Proof. Let $\phi_{j}$ be a Cauchy sequence in $F$. Clearly, there exists $\phi \in \mathbf{C}(-\infty, 0]$ such that $\phi_{j}$ converges to $\phi$ uniformly on every compact set of $(-\infty, 0]$. Consider the Banach space $l^{1}\left(\mathbf{C}\left(\left[0, k \tau_{1}\right]\right)\right)$. For every $j \in \mathbb{N}$, define $G_{j} \in l^{1}\left(C\left(\left[0, k \tau_{1}\right]\right)\right)$ as

$$
G_{j}^{(i)}(s)=b_{n(k)+(i-1)} \phi_{j}\left(s-\tau_{n(k)+(i-1)}\right), \quad i \in \mathbb{N} .
$$

By the hypothesis, $G_{j}$ is a Cauchy sequence in $l^{1}\left(\mathbf{C}\left(\left[0, k \tau_{1}\right]\right)\right)$ and hence converges to some $G \in l^{1}\left(\mathbf{C}\left(\left[0, k \tau_{1}\right]\right)\right)$. For fixed $i$ and $s, \lim _{j \rightarrow \infty} G_{j}^{(i)}(s)=G^{(i)}(s)$ and hence $G^{(i)}(s)=$ $b_{n(k)+(i-1)} \phi\left(s-\tau_{n(k)+(i-1)}\right)$. This implies that $p_{k}(\phi)<\infty$ and $\phi_{j}$ converges to $\phi$ in $F$. The proof is complete.

Now we state the definition of a mild solution of an abstract Cauchy problem in Frechet spaces from [4].

Let $X$ be a Frechet space whose topology is given by the family of seminorms $\left\{q_{k}: k \in \mathbb{N}\right\}$ and $\mathcal{D}(A)$ be a subset of $X$. For a closed operator $A: \mathcal{D}(A) \longrightarrow X$ and $\phi \in X$, consider the abstract Cauchy problem

$$
\begin{aligned}
\frac{\mathrm{d} u}{\mathrm{~d} t} & =A u, \\
u(0) & =\phi .
\end{aligned}
$$

\section{DEFINITION 1.12}

A function $u(\cdot) \in \mathbf{C}([0, \infty), \mathcal{D}(A)) \cap \mathbf{C}^{1}([0, \infty), X)$ that satisfies (1.3) is said to be a solution of Problem (1.3). A function $u(\cdot) \in \mathbf{C}([0, \infty), X)$ is said to be a mild solution of (1.3), if $v(t)=\int_{0}^{t} u(s) \mathrm{d} s \in \mathcal{D}(A)$, for all $t \geq 0$, and

$$
\frac{\mathrm{d}(v(\cdot))}{\mathrm{d} t}(t)=A(v(t))+\phi, \quad t \geq 0 .
$$

\section{Main results}

Let $F$ be as in the previous section. Define $\mathcal{D}(A)=\left\{\varphi \in F: \varphi^{\prime} \in F\right.$ and $\left.\varphi^{\prime}(0)=L \varphi\right\}$ and $A: \mathcal{D}(A) \longrightarrow F$ as $A \varphi=\varphi^{\prime}$ where

$$
L \varphi=a \varphi(0)+\sum_{i=1}^{\infty} b_{i} \varphi\left(-\tau_{i}\right) .
$$

\section{DEFINITION 2.1}

We say that a function $x: \mathbb{R} \longrightarrow \mathbb{R}$ is a solution to (1.1) if the following hold:

(i) $x$ is continuous and $x(\theta)=\phi(\theta)$ for all $\theta \in(-\infty, 0]$.

(ii) The restriction of $x$ to $[0, \infty)$ is continuously differentiable.

(iii) $x^{\prime}(t)=a x(t)+\sum_{i=1}^{\infty} b_{i} x\left(t-\tau_{i}\right)$ for all $t \geq 0$.

Remark 2.2. Note that our definition of the solution does not imply that $x$ is differentiable from the left at $t=0$. 
Theorem 2.3. Let $F, L$ and $A$ be as in the beginning of this section. Then A generates a strongly continuous semigroup $\left\{S_{t}: t \geq 0\right\}$ of bounded linear operators on F. Further, for a given $\phi \in F$, the map $x: \mathbb{R} \rightarrow \mathbb{R}$ defined as

$$
\begin{aligned}
& x(t)=\phi(t), \quad t \in(-\infty, 0], \\
& x(t)=\left[S_{t} \phi\right](0), \quad t \in(0, \infty)
\end{aligned}
$$

is a unique solution to (1.1).

Besides, fixing $\phi \in F$ and defining $u:[0, \infty) \rightarrow F$ as $u(t)=S_{t} \phi, u(\cdot)$ is a mild solution to the abstract Cauchy problem (1.3).

We need the following lemmas to prove Theorem 2.3 and we actually define the semigroup via the solution to (1.1).

Lemma 2.4. Let $\phi \in F$. The problem (1.1) has a unique solution $x: \mathbb{R} \rightarrow \mathbb{R}$.

Further, for each $k \in \mathbb{N}$ there exists a finite subset $\Lambda$ of $\left\{\|\cdot\|_{k}: k \in \mathbb{N}\right\} \cup\left\{p_{k}: k \in \mathbb{N}\right\}$ and a constant $C_{k} \geq 0$ such that

$$
\sup \left\{|x(s)|: s \in\left[0, k \tau_{1}\right]\right\} \leq C_{k} \max \{q(\phi): q \in \Lambda\} .
$$

Proof. Consider $t \in\left[0, \tau_{1}\right]$. Clearly, $t-\tau_{i} \leq t-\tau_{1}$ for all $i \in \mathbb{N}$. Thus for $t \in\left[0, \tau_{1}\right], t-$ $t_{i} \leq 0$. Define $y_{1}:\left[0, \tau_{1}\right] \rightarrow \mathbb{R}$ as the unique solution of the initial value problem.

$$
\begin{aligned}
& x^{\prime}(t)=\operatorname{ax}(t)+\sum_{i=1}^{\infty} b_{i} \phi\left(t-\tau_{i}\right), \\
& x(0)=\phi(0) .
\end{aligned}
$$

Note that as $\phi \in F, t \rightarrow \sum_{i=1}^{\infty} b_{i} \phi\left(t-\tau_{i}\right)$ defines a continuous function on [0, $\left.\tau_{1}\right]$. We have

$$
y_{1}(t)=\phi(0) \mathrm{e}^{a t}+\mathrm{e}^{a t} \int_{0}^{t} \mathrm{e}^{-a s}\left(\sum_{i=1}^{\infty} b_{i} \phi\left(s-\tau_{i}\right)\right) \mathrm{d} s .
$$

Define $x_{1}:\left(-\infty, \tau_{1}\right] \rightarrow \mathbb{R}$ as

$$
\begin{aligned}
x_{1}(s) & =\phi(s), \quad s \in(-\infty, 0] \\
& =y_{1}(s), \quad s \in\left[0, \tau_{1}\right] .
\end{aligned}
$$

In the remaining part of the proof we shall assume that $a \neq 0$. The estimates for $a=0$ are easier to obtain. Clearly,

$$
\sup \left\{\left|x_{1}(t)\right|: t \in\left[0, \tau_{1}\right]\right\} \leq\left(\sup _{s \in\left[0, \tau_{1}\right]} \mathrm{e}^{a s}\right)\|\phi\|_{1}+\left(\sup _{s \in\left[0, \tau_{1}\right]} \frac{\mathrm{e}^{a s}-1}{a}\right) p_{1}(\phi) .
$$

Here, note that for $r>0, \frac{\mathrm{e}^{a r}-1}{a}>0$ for all $a \neq 0$. Now we claim that for each $k \in \mathbb{N}$, there exists a function $x_{k}:\left(-\infty, k \tau_{1}\right] \rightarrow \mathbb{R}$ with the following properties: 
(i) For each $t \in\left[0, k \tau_{1}\right]$,

$$
\sum_{i=1}^{\infty} b_{i} x_{k}\left(t-\tau_{i}\right)
$$

converges and this summation defines a continuous function on [0,k $\left.\tau_{1}\right]$.

(ii) $x_{k}$ is the unique solution to

$$
\begin{aligned}
& x^{\prime}(t)=a x(t)+\sum_{i=1}^{\infty} b_{i} x\left(t-\tau_{i}\right), \quad \text { for } t \in\left[0, k \tau_{1}\right] \\
& x(\theta)=\phi(\theta), \quad \text { for } t \in(-\infty, 0] .
\end{aligned}
$$

(iii) There exists a finite subset $\Lambda$ of $\left\{\|\cdot\|_{k}: k \in \mathbb{N}\right\} \cup\left\{p_{k}: k \in \mathbb{N}\right\}$ and a constant $C_{k} \geq 0$ such that

$$
\sup \left\{\left|x_{k}(s)\right|: s \in\left[0, k \tau_{1}\right]\right\} \leq C_{k} \max \{q(\phi): q \in \Lambda\} .
$$

We prove this by induction on $k$. The case $k=1$ is already proved.

Assuming that our claim is true for arbitrary $k \in \mathbb{N}$, we show that the claim is true for $k+1$. Define $I_{k}=\left[(k-1) \tau_{1}, k \tau_{1}\right]$ and $\left\|x_{k}\right\|_{I_{k}}$ as

$$
\left\|x_{k}\right\|_{I_{k}}=\sup \left\{\left|x_{k}(s)\right|: s \in\left[(k-1) \tau_{1}, k \tau_{1}\right]\right\} .
$$

For $s \in I_{k+1}$ and $i \in \mathbb{N}, s-\tau_{i} \in\left(-\infty, k \tau_{1}\right]$ and so we can consider the summation

$$
\sum_{i=1}^{\infty} b_{i} x_{k}\left(s-\tau_{i}\right)=\sum_{i=1}^{n(k+1)-1} b_{i} x_{k}\left(s-\tau_{i}\right)+\sum_{i=n(k+1)}^{\infty} b_{i} x_{k}\left(s-\tau_{i}\right) .
$$

The first summation involves only finitely many terms. For $s \in\left[k \tau_{1},(k+1) \tau_{1}\right]$ and $i \geq n(k+1), s-\tau_{i}<s-(k+1) \tau_{1} \leq 0$ and hence

$$
\sum_{i=n(k+1)}^{\infty} b_{i} x_{k}\left(s-\tau_{i}\right)=\sum_{i=n(k+1)}^{\infty} b_{i} \phi\left(s-\tau_{i}\right) .
$$

So, for $s \in I_{k+1}$, the summation $\sum_{i=1}^{\infty} b_{i} x_{k}\left(s-\tau_{i}\right)$ defines a continuous function on $I_{k+1}$.

For a given $k \in \mathbb{N}-\{1\}$, define $m(k)$ as the smallest positive integer such that

$$
-m(k)<\min \left\{(k-1) \tau_{1}-\tau_{i}: i=1,2, \ldots, n(k)-1\right\} .
$$

The following estimates follow from the definition of the seminorms and the integers $m(k)$ :

$$
\sup \left\{\left|\sum_{i=n(k+1)}^{\infty} b_{i} x_{k}\left(s-\tau_{i}\right)\right|: s \in\left[k \tau_{1},(k+1) \tau_{1}\right]\right\} \leq p_{k+1}(\phi) .
$$

Moreover,

$$
\begin{aligned}
& \sup \left\{\left|\sum_{i=n}^{n(k+1)-1} b_{i} x_{k}\left(s-\tau_{i}\right)\right|: s \in\left[k \tau_{1},(k+1) \tau_{1}\right]\right\} \\
& \leq \sum_{i=1}^{n(k+1)-1}\left|b_{i}\right| \times \max \left\{\|\phi\|_{m(k+1)},\left\|x_{k}\right\|_{I_{k}}\right\} .
\end{aligned}
$$


Define $y_{k+1}:\left[k \tau_{1},(k+1) \tau_{1}\right] \rightarrow \mathbb{R}$ as

$$
y_{k+1}(t)=x_{k}\left(k \tau_{1}\right) \mathrm{e}^{a\left(t-k \tau_{1}\right)}+\mathrm{e}^{a t} \int_{k \tau_{1}}^{t} \mathrm{e}^{-a s}\left(\sum_{i=1}^{\infty} b_{i} x_{k}\left(s-\tau_{i}\right)\right) \mathrm{d} s .
$$

From (2.5) and (2.6), we get the estimate

$$
\begin{aligned}
\sup & \left\{\left|\sum_{i=1}^{\infty} b_{i} x_{k}\left(s-\tau_{i}\right)\right|: s \in\left[k \tau_{1},(k+1) \tau_{1}\right]\right\} \\
& \leq\left[\sum_{i=1}^{n(k+1)-1}\left|b_{i}\right| \times \max \left\{\|\phi\|_{m(k+1)},\left\|x_{k}\right\|_{I_{k}}\right\}+p_{k+1}(\phi)\right] .
\end{aligned}
$$

Defining $x_{k+1}:\left(-\infty,(k+1) \tau_{1}\right] \rightarrow \mathbb{R}$ as

$$
\begin{aligned}
x_{k+1}(s) & =x_{k}(s), s \in\left(-\infty, k \tau_{1}\right] \\
& =y_{k+1}(s), s \in\left(k \tau_{1},(k+1) \tau_{1}\right],
\end{aligned}
$$

our claims (i) and (ii) are proved. Now, we proceed to prove (iii) for $k+1$. From the definition of $y_{k+1}$ and the estimate (2.7), we get, for $k \in \mathbb{N}$,

$$
\begin{aligned}
\sup \left\{\left|x_{k+1}(s)\right|: s \in\left[k \tau_{1},(k+1) \tau_{1}\right]\right\}=\left\|x_{k+1}\right\|_{I_{k+1}} \\
=\sup \left\{\left|y_{k+1}(s)\right|: s \in\left[k \tau_{1},(k+1) \tau_{1}\right]\right\} \\
\leq\left(\sup _{s \in\left[0, k \tau_{1}\right]} \mathrm{e}^{a s}\right)\left\|x_{k}\right\|_{I_{k}}+\left(\sup _{s \in\left[0, \tau_{1}\right]} \frac{\mathrm{e}^{a(k+1) s}-1}{a}\right) \\
\quad \times\left[\sum_{i=1}^{n(k+1)-1}\left|b_{i}\right| \times \max \left\{\|\phi\|_{m(k+1)},\left\|x_{k}\right\|_{I_{k}}\right\}+p_{k+1}(\phi)\right] .
\end{aligned}
$$

Since

$$
\left\|x_{k}\right\|_{I_{k}} \leq \sup \left\{\left|x_{k}(s)\right|: s \in\left[0, k \tau_{1}\right]\right\}
$$

the assertion (iii) for $k+1$ follows from the estimate (2.8) and hence the assertion (iii) for $k$.

The solution to (1.1) is obtained by patching the functions $x_{k}$. Uniqueness of $x$ now follows.

Lemma 2.5. Let $\varphi \in F$ and $x: \mathbb{R} \rightarrow \mathbb{R}$ be a continuous function such that $x(\theta)=\varphi(\theta)$ for all $\theta \in(-\infty, 0]$. Define $u:[0, \infty) \rightarrow \mathbf{C}((-\infty, 0])$ as $[u(t)](\theta)=x(t+\theta)$. Then $u \in \mathbf{C}([0, \infty) ; F)$.

Proof. Fix $t \in\left[0, j \tau_{1}\right]$. It is trivial to check that $u(t) \in \mathbf{C}((-\infty, 0])$. We also have the estimate

$$
\begin{aligned}
\|u(t)\|_{k} & =\sup \{\theta \in[-k, 0]:|[u(t)](\theta)|\} \\
& \leq \max \left(\|\varphi\|_{k}, \sup \left\{|x(t)|: t \in\left[0, j \tau_{1}\right\}\right) .\right.
\end{aligned}
$$


Now we show that $p_{k}(u(t))<\infty$ for each $k \in \mathbb{N}$. Let $s \in\left[0, k \tau_{1}\right]$ and $t \in\left[0, j \tau_{1}\right]$. It is clear that $n(k+j) \geq n(k)$ and we have the following assertions:

$$
i \geq n(k) \Rightarrow\left(s-\tau_{i}\right) \leq 0
$$

and

$$
n(k) \leq i<n(k+j) \Rightarrow-(k+j) \tau_{1} \leq t+s-\tau_{i} \leq j \tau_{1} .
$$

Thus, for every $K \geq n(k+j)$, we have

$$
\begin{aligned}
\sum_{i=n(k)}^{K} b_{i}[u(t)]\left(s-\tau_{i}\right)= & \sum_{i=n(k)}^{n(k+j)-1} b_{i}[u(t)]\left(s-\tau_{i}\right) \\
& +\sum_{i=n(k+j)}^{K} b_{i}[u(t)]\left(s-\tau_{i}\right) \\
= & \sum_{i=n(k)}^{n(k+j)-1} b_{i} x\left(t+s-\tau_{i}\right) \\
& +\sum_{i=n(k+j)}^{K} b_{i} \phi\left(t+s-\tau_{i}\right) .
\end{aligned}
$$

Now choosing a positive integer $m \geq(k+j) \tau_{1}$, we have the estimate

$$
p_{k}(u(t)) \leq \sum_{i=1}^{n(k+j)}\left|b_{i}\right| \max \left(\sup \left\{|x(t)|: t \in\left[0, j \tau_{i}\right\},\|\phi\|_{m}\right)+p_{(k+j)}(\phi) .\right.
$$

Equations (2.9) and (2.10) show that $u(t) \in F$.

Next, we show the continuity of $u$ at an arbitrary $t_{0} \in[0, \infty)$. Let $\epsilon>0$ be given. By the uniform continuity of $x$ on $\left[-k-1+t_{0}, t_{0}+1\right]$, there exists $\delta>0$ such that for $p, q \in\left[-k-1+t_{0}, t_{0}+1\right]$ with $|p-q|<\delta,|x(p)-x(q)|<\epsilon$. Take $\delta^{*}=\min (1, \delta)$ and consider $s$ with $\left|t_{0}-s\right|<\delta^{*}$. Clearly, for any $\theta \in[-k, 0], t_{0}+\theta$ and $s+\theta$ both belong to $\left[-k-1+t_{0}, t_{0}+1\right]$ and hence

$$
\sup \left\{\left|x\left(t_{0}+\theta\right)-x(s+\theta)\right|: \theta \in[-k, 0]\right\}<\epsilon .
$$

That is, given $\epsilon>0$, there is a $\delta^{*}>0$ such that

$$
\left\|u(s)-u\left(t_{0}\right)\right\|_{k}<\epsilon
$$

whenever $\left|s-t_{0}\right|<\delta^{*}$.

Next, we claim that for a given $\epsilon>0$ and $t_{0} \in\left[0, j \tau_{1}\right]$, there exists $\delta>0$ such that $p_{k}\left(u\left(t_{0}\right)-u(t)\right)<\epsilon$ whenever $t_{0} \in\left[0, j \tau_{1}\right]$ satisfies $\left|t-t_{0}\right|<\delta$. 
By Proposition 1.6, and the definition of $F$, for a given $\varphi \in F, G_{k}:\left[0, k \tau_{1}\right] \rightarrow l^{1}(\mathbb{R})$ defined as $\left[G_{k}(s)\right]^{(i)}=b_{n(k)+(i-1)} \varphi\left(s-\tau_{n(k)+(i-1)}\right)$ is a continuous function. Consider

$$
\begin{aligned}
\sum_{i=n(k)}^{K} b_{i}\left[u\left(t_{0}\right)-u(t)\right]\left(s-\tau_{i}\right)= & \sum_{i=n(k)}^{n(k+j)-1} b_{i}\left[u\left(t_{0}\right)-u(t)\right]\left(s-\tau_{i}\right) \\
& +\sum_{i=n(k+j)}^{K} b_{i}\left[u\left(t_{0}\right)-u(t)\right]\left(s-\tau_{i}\right) \\
= & \sum_{i=n(k)}^{n(k+j)-1} b_{i}\left[x\left(t_{0}+s-\tau_{i}\right)-x\left(t+s-\tau_{i}\right)\right] \\
= & \sum_{i=n(k+j)}^{K} b_{i}\left[\phi\left(t_{0}+s-\tau_{i}\right)-\phi\left(t+s-\tau_{i}\right)\right]
\end{aligned}
$$

and hence

$$
\begin{aligned}
\sum_{i=n(k)}^{\infty} b_{i}\left[u\left(t_{0}\right)-u(t)\right]\left(s-\tau_{i}\right) & =\sum_{i=n(k)}^{n(k+j)-1} b_{i}\left[x\left(t_{0}+s-\tau_{i}\right)-x\left(t+s-\tau_{i}\right)\right] \\
& +\sum_{i=n(k+j)}^{\infty} b_{i}\left[\phi\left(t_{0}+s-\tau_{i}\right)-\phi\left(t+s-\tau_{i}\right)\right] .
\end{aligned}
$$

By the uniform continuity of $G_{n(k+j)}$ on [0, $\left.(k+j) \tau_{1}\right]$, given $\epsilon>0$, there exists a $\delta_{1}>0$ such that $|p-q|<\delta_{1}$ implies that

$$
\sum_{i=n(k+j)}^{\infty}\left|b_{i}\right|\left|\left[\phi\left(p-\tau_{i}\right)-\phi\left(q-\tau_{i}\right)\right]\right|<\epsilon / 2 .
$$

So, for $t_{0}$ and $t \in\left[0, j \tau_{1}\right]$ with $\left|t_{0}-t\right|<\delta_{1}$,

$$
\sup \left\{\sum_{i=n(k+j)}^{\infty}\left|b_{i}\right|\left|\left[\phi\left(p-\tau_{i}\right)-\phi\left(q-\tau_{i}\right)\right]\right|: s \in\left[0, k \tau_{1}\right]\right\}<\epsilon / 2 .
$$

Since the expression $\sum_{i=n(k)}^{n(k+j)-1} b_{i}\left[x\left(t_{0}+s-\tau_{i}\right)-x\left(t+s-\tau_{i}\right)\right]$ involves evaluation of $x$ over a compact set, given $\epsilon>0$, there exists $\delta_{2}>0$ such that

$$
\sum_{i=n(k)}^{n(k+j)-1} \sup \left\{\left|b_{i}\right|\left|\left[x\left(t_{0}+s-\tau_{i}\right)-x\left(t+s-\tau_{i}\right)\right]\right|\right\}<\epsilon / 2
$$

whenever $t_{0}, t \in\left[0, j \tau_{1}\right]$ and $\left|t_{0}-t\right|<\delta_{2}$. Our claim is now proved by taking $\delta=$ $\min \left(\delta_{1}, \delta_{2}\right)$.

Proof of Theorem 2.3. By Lemma 2.4, for a given $\phi \in F$, we have a solution $x_{\phi}: \mathbb{R} \rightarrow \mathbb{R}$ to $(1.1)$. 
Define $S_{t}: F \rightarrow F$ as

$$
\begin{aligned}
{\left[S_{t} \phi\right](\theta) } & =x_{\phi}(t+\theta), \quad t+\theta>0 \\
& =\phi(t+\theta), \quad t+\theta \leq 0 .
\end{aligned}
$$

Lemma 2.5 shows that $S_{t} \phi \in F$ for all $t \geq 0$ and the map $u(t)=S_{t} \phi$ is a continuous function from $[0, \infty)$ into $F$. From the definition of $S_{t}$ one can verify linearity of each $S_{t}$ and the property $S_{t+s}=S_{t} S_{s}$. We need only to check that each $S_{t}$ is a bounded linear map on $F$. This follows from the estimates (2.9), 2.10) and (2.1)

Next, we have

$$
\begin{aligned}
& {[u(t)](\theta)=\phi(t+\theta), \quad t+\theta \leq 0} \\
& {[u(t)](\theta)=\phi(0)+\int_{0}^{t+\theta} L(u(s)) \mathrm{d} s, \quad t+\theta>0}
\end{aligned}
$$

and using (2.11), it is easy to see that $u$ is indeed a mild solution to (1.3).

The Banach space $\mathbf{C}_{g}$ defined below was the phase space used in [4] for the study of infinite delay equations of which (1.1) is a special case. An interesting observation is that $F$ contains $\mathbf{C}_{g}$.

Example 2.6. Let $g:(-\infty, 0] \rightarrow[1, \infty)$ be a continuous non-increasing function such that

$$
\sum_{i=1}^{\infty} b_{i} g\left(-\tau_{i}\right)<\infty
$$

Consider the space $\mathbf{C}_{g}$ of all $\varphi \in \mathbf{C}(-\infty, 0] \rightarrow \mathbb{R}$ such that

$$
\|\varphi\|_{g} \stackrel{\text { def }}{=} \sup \left\{\frac{|\varphi(\theta)|}{g(\theta)}: \theta \in(-\infty, 0]\right\}<\infty \text {. }
$$

Then $\mathbf{C}_{g} \subset F$.

Proof. Consider $\left|b_{i} \| \varphi\left(s-\tau_{i}\right)\right|=\left|b_{i}\right| \frac{\left|\varphi\left(s-\tau_{i}\right)\right|}{g\left(s-\tau_{i}\right)} g\left(s-\tau_{i}\right)$. For $s \in\left[0, k \tau_{1}\right]$ and $i \geq n(k), 0 \geq$ $s-\tau_{i} \geq-\tau_{i}$ and hence

$$
\sup \left\{\left|b_{i} \| \varphi\left(s-\tau_{i}\right)\right|: s \in\left[0, k \tau_{1}\right]\right\} \leq\left(\|\phi\|_{g}\right)\left|b_{i}\right| g\left(-\tau_{i}\right) .
$$

Now, the assertion follows from (2.12).

\section{Acknowledgement}

Major part of this work was done while the author was with the Department of Mathematics, Indian Institute of Science, Bangalore. The author acknowledges the referee whose comments greatly improved the presentation of the paper and who pointed out many misprints. 


\section{References}

[1] Atkinson F V and Haddock J R, On determining phase spaces for functional differential equations, Funkcialaj Ekvacioj 31 (1988) 331-347

[2] Burger R, An integro-differential equation from population genetics and perturbations of differentiable semigroups in Frechet spaces, Proc. R. Soc. Edin. 118A (1991) 63-73

[3] Choe Y H, $C_{0}$-semigroups on a locally convex space, J. Math. Anal. Appl. 106 (1985) 293-320

[4] de Laubenfels R, Existence families, functional calculi and evolution equations, LNM 1570 (Springer Verlag) (1994)

[5] Haddock J R, Nkashama M N and Wu J, Asymptotic constancy for linear neutral Volterra integrodifferential equations, Tohuku Math. J. 41 (1989) 689-710

[6] Haddock J and Terjeki J, On the location of positive limit sets for autonomous functional differential equations with infinite delay, J. Diff. Eqns 86 (1990) 1-31

[7] Hale J K and Verduyn Lunel M, Introduction to functional differential equations (New York: Springer Verlag) (1993)

[8] Hale J K and Kato J, Phase spaces for retarded equations with infinite delay, Funkcial Ekvac. 21 (1978) 11-41

[9] Hamilton R, The inverse function theorem of Nash and Moser, Bull. Am. Math. Soc. 7 (1982) 1-64

[10] Kantorovitz S, The Hille-Yosida space of an arbitrary operator, J. Math. Anal. Appl. 136 (1988) 107-111

[11] Komura T, Semigroups of operators in locally convex spaces, J. Funct. Anal. 2 (1968) 258-296

[12] Lobanov S G and Smolyanov O G, Ordinary differential equations in locally convex spaces, Usp. Mat. Nauk. 49 (1994) 93-168

[13] Oharu S, Semilinear evolution equations in Frechet spaces, Differential equations in Banach spaces (Springer Verlag) LNM 1223 (1986) pp. 186-207

[14] Rudin W, Functional Analysis (New Delhi: TMH) (1974) 